\title{
SPOROGONIE ET SCHIZOGONIE PRÉ-ÉRYTHROCYTAIRE DE PLASMODIES DE LÉMURIENS MALGACHES ${ }^{1}$
}

\author{
L. RABETAFIKA*, I. LANDAU**, D. BACCAM** \\ J.-P. LEPERS***, P. COULANGES***, S. RAKOTOFIRINGA*
}

RÉSUMÉ. Une étude expérimentale du cycle des Plasmodiums hébergés par un Lemur macaco macaco a abouti aux résultats suivants :

Trois des quatre espèces présentes $(P$. coulangesi, $P$. percygarnhami et $P$. bucki) ont été transmises par Anopheles stephensi à un Lemur macaco macaco sain. La quatrième espèce (?) Plasmodium lemuris, n'est pas apparue chez le nouvel hôte, ce qui est en faveur de l'hypothèse (Landau et coll., 1989) selon laquelle il ne s'agirait pas d'un Plasmodium mais plutôt de Nyctheria.

Les différents stades de la sporogonie et de la schizogonie hépatique sont décrits, mais il n’a pas été possible de les identifier de façon spécifique.

Sporogonie : A $25^{\circ} \mathrm{C}$ les oocystes mûrissent entre 13 et 15 jours. Les tailles moyennes des oocystes mûrs mesurés dans cinq cages de Moustiques différentes varient entre $52 \mu \mathrm{m}$ et $61 \mu \mathrm{m}$. Les sporozoïtes sont nombreux dans les glandes salivaires à partir du $15^{\mathrm{e}}$ ou $\mathrm{du} 16^{\mathrm{e}}$ jour. Ils mesurent $8,5 \mu \mathrm{m} \pm 0,12$.

Schizogonie hépatique : Un Lemur fulvus fulvus mayotensis des Commores a été inoculé de façon massive avec des sporozoïtes, pour l'étude des stades exo-érythrocytaires. La durée de la période prépatente a été de 9 jours. Deux populations de schizontes, l'une présentant des pseudocytomères, l'autre non, sont observées dans le foie.

Mots-clés : Plasmodium. Lémuriens malgaches. Transmission. Sporogonie. Shizogonie préérythrocytaire.

\section{Sporogony and pre-erythrocytic schizogony of Plasmodia from Lemurs of Madagascar.}

SUMMARY. Experimental studies on the life-cycle of Plasmodia from Lemur macaco macaco gave the following results:

Three out of the four species parasitizing this host were transmitted, through Anopheles stephensi, to a clean Lemur macaco macaco. The fourth species, (?) Plasmodium lemuris, did not show in the new host and this strengthens Landau et al. (1989) belief that this species is not a true Plasmodium but in fact a Nyctheria.

The various stages of sporogony and hepatic schizogony are described but the authors were unable to identify them specifically.

1. Travail ayant bénéficié de l'appui du Programme Spécial/PNUD/Banque Mondiale/OMS de Recherche et de Formation concernant les Maladies Tropicales.

* Laboratoire de Zoologie, Université de Madagascar, Antananarivo, Madagascar.

** Laboratoire de Protozoologie et Parasitologie comparée, EPHE et Laboratoire de Zoologie (Vers) associé au CNRS, Muséum National d'Histoire Naturelle, 61, rue Buffon, 75231 Paris Cedex 05.

*** Institut Pasteur de Madagascar, Boite Postale 1274, Antanarivo, Madagascar.

Accepté le 13 février 1988. 
Sporogony: At $25^{\circ} \mathrm{C}$, oocysts mature in 13 to 15 days. Mean sizes of mature oocysts, in Mosquitoes from 5 different cages, varied between $52 \mu \mathrm{m}$ and $61 \mu \mathrm{m}$. Sporozoites were numerous in the salivary glands from day 15 . They are $8,5 \pm 0,12$ long.

Hepatic schizogony: A Lemur fulvus fulvus mayottensis from Commores was massively inoculated with sporozoites, in order to study exo-erythrocytic stages. The prepatent period lasted 9 days. Two populations of schizonts, one with and one without pseudocytomeres, where seen in the liver.

Key-words: Plasmodium. Lemurs. Madagascar. Transmission. Sporogony. Pre-erythrocytic schizogony.

\section{Matériel et méthodes}

\section{1 - Matériel biologique et chronologie des expériences}

$a$ - Espèces plasmodiales

Le Lémur 38CH (cf. ci-dessous), naturellement infecté, hébergeait quatre espèces : Plasmodium coulangesi Lepers et coll., 1989, Plasmodium percygarnhami Landau et coll., 1989, Plasmodium bucki Landau et coll. 1989 et (?) Plasmodium lemuris Huff et Hoogstral, 1963.

$b$ - Lémuriens

1 - Lemur macaco macaco L., 1766.

Deux Lémuriens provenant de la région de Ambanja, au Nord de Madagascar ont été utilisés.

- Lemur mâle no $38 C H$ : maintenu en captivité à l'Institut Pasteur de Madagascar depuis novembre 1985; faiblement infecté à son arrivée (parasitémie de 0,02\%) le 4.12.1987; splénectomisé le 9.12.1987.

Après la splénectomie la parasitémie a augmenté rapidement. Les Anophèles ont été gorgés les 14.12.1987, 16.12 .1987 et 17.12.1987 (respectivement J5, J7 et J8 après la splénectomie), à un moment ou les gamétocytes étaient abondants.

Le 19.12.1987, le taux de parasitémie ayant atteint $10 \%$, l'animal a été traité pendant trois jours par la quinine; il devient apparemment négatif.

A partir du 5.1.1988 (J17 après le traitement) une recrudescence s'amorce. Le 13.1.1988 et le 14.1.1988 la parasitémie ayant atteint $5 \%$ et des gamétocytes étant présents, de nouveaux Anophèles sont gorgés.

Le 18.1.1988, la parasitémie étant élevée $(10 \%)$ un nouveau traitement par la quinine est effectué. L’infection redevient inapparente.

- Lemur femelle no 39 CH : maintenu en captivité au parc Zoologique de Tsimbazaza depuis novembre 1983 ; arrivé au Muséum, comme le précédent le 4.12.1988 ; deux frottis effectués, l'un le jour de son arrivée, l'autre 21 jours plus tard sont négatifs. 
Le 4.1.1988 et le 6.1.1988 il est inoculé par voie intra-veineuse avec des sporozoïtes obtenus par dissection de glandes salivaires de 270 Anophèles gorgés sur le Lémur 38CH.

Le 11.1.1988 (J7 après la première injection) une biopsie de foie est pratiquée.

Les premiers parasites sanguins apparaissent le 13.1.1988 (J9 après la première injection). Les 20.1.1988 et 21.1.1988 des Anophèles sont gorgés, puis l'animal est traité par la quinine.

2 - Lemur fulvus fulvus mayottensis Schlegel, 1826, originaire des Commores; présente une tumeur de la lèvre supérieure. Il arrive au laboratoire le 29.1.1988; le frottis de sang est négatif et il reçoit ce jour, par voie intra-veineuse, un premier broyat de 249 thorax et trois jours plus tard, un deuxième broyat de 240 thorax d'Anopheles stephensi gorgés sur Lemur macaco 39GH. Une autopsie est effectuée le 7.2.1988 (J9 après la première inoculation).

3 - Microcebus murinus murinus Miller, 1977 provenant de l'élevage de Arlette Petter.

Il reçoit par voie intra-péritonéale le 6.1.1988 et le 5.2.1988 des sporozoïtes provenant respectivement d'Anophèles gorgés sur 38 et $39 \mathrm{CH}$. Des frottis ont été effectués régulièrement, au moins une fois par semaine jusqu'au 19.3.1988.

$c$ - Rongeurs

Différents Rongeurs sont inoculés par voie I. V. ou I. P. avec du sang du Lémur 38CH : 10 Souris blanches, dont 7 splénectomisées ; 4 Rats blancs dont 2 splénectomisés; 1 Cobaye splénectomisé ; 5 Hamsters dont 3 splénectomisés.

Trois jeunes Rats et une Souris blanche sont également inoculés en I. V. avec des sporozoïtes.

Ils ont été examinés au moins une fois par semaine pendant 2 mois.

$d$ - Vecteurs expérimentaux

Ce sont des Anopheles stephensi d'origine indienne, élevés au laboratoire, issus d'un élevage établi par Shute et Maryon (1966) en Angleterre.

Ils sont maintenus à une température de $24-25^{\circ} \mathrm{C}$.

2 - Étude du GyGle

Le gorgement des Anophèles est effectué sur Lémur endormi avec un mélange d'Imalgène et de Vetranquil (20\%).

Les glandes salivaires d'Anophèles sont disséquées, ou les thorax broyés, dans du RPMI froid, additionné de $10 \%$ de sérum de Veau fœtal et, après broyage, sont inoculées par voie IV.

Les fixations de foie de Lemur et des Moustiques sont faites par le Carnoy et les coupes colorées par le Giemsa-colophane. 


\section{Résultats}

\section{1 - Transmission eXPérimentale}

Aucune parasitémie n'a été observée cbez le Microcèbe et les Rongeurs inoculés.

- Le Lémur $39 \mathrm{CH}$ sain a présenté, après son infestation avec des Anophèles gorgés sur $38 \mathrm{CH}$, une parasitémie avec 3 des quatre espèces observées chez ce dernier : $P$. coulangesi, $P$. percygarnhami et $P$. bucki. (?) $P$. lemuris n'a pas été transmis.

Les premiers parasites ont été décellés 9 jours après la première injection de sporozoïtes. Rappelons que l'animal avait reçu deux injections de sporozoïtes à 48 heures d'intervalle. Le déroulement de l'infection sanguine chez cet hôte a été décrit ailleurs (Landau et coll., 1989).

- Le Lemur fulvus infecté à partir d'Anophèles gorgés sur 39CH est également devenu positif dans le sang, 9 jours après la première injection de sporozoïtes.

\section{2 - Sporogonie}

Des ookinètes ont été observés dans des étalements de contenu intestinal d'Anophèles gorgés depuis 24 heures. Nous observons également des ookinètes, installés sous la basale à l'extérieur de l'épithélium intestinal, en coupes d'estomacs d'Anophèles gorgés depuis 6 jours ; ils coexistent avec des oocystes plurinucléés.

Les oocystes ont été plus nombreux dans les cages gorgées sur le Lémur infecté expérimentalement (de 20 à 50 oocystes par Moustique) que dans celui splénectomisé après une infection naturelle (de 2 à 25 oocystes).

Les premiers oocystes mûrs (fig. 1), sont observés dès le $11^{\mathrm{e}}$ jour, mais la majorité mûrit entre 13 et 15 jours.

Les tailles moyennes des oocystes mûrs mesurés à frais, entre lame et lamelle, varient peu en fonction de l'animal et du jour de gorgement (de 52 à $61 \mu \mathrm{m}$ ). En effet, les moyennes des diamètres d'oocystes mesurés dans 5 cages d'Anophèles gorgés à différents moments de l'infection et sur deux lémuriens est : $52 \pm 1 \mu \mathrm{m}$, $53 \pm 1,2 \mu \mathrm{m}, 55 \pm 0,8 \mu \mathrm{m}, 56 \pm 1,6 \mu \mathrm{m}, 61 \pm 2,2 \mu \mathrm{m}$.

En coupes histologiques de Moustique (fig. 2, 1), ils apparaissent comme des masses ovalaires dans lesquelles on note un cytoplasme formant un ruban sinueux bleu, très chromophile ; les sporozoïtes sont disposés en faisceaux parallèles le long des bords du cytoplasme.

Les sporozoïtes peuvent faire leur première apparition dans les glandes salivaires dès le $12^{\mathrm{e}}$ jour mais ils ne sont nombreux qu'à partir des $15^{\mathrm{e}}, 16^{\mathrm{e}}$ jours. Là encore ils sont beaucoup plus nombreux dans les Moustiques gorgés sur le Lémur infecté expérimentalement. 

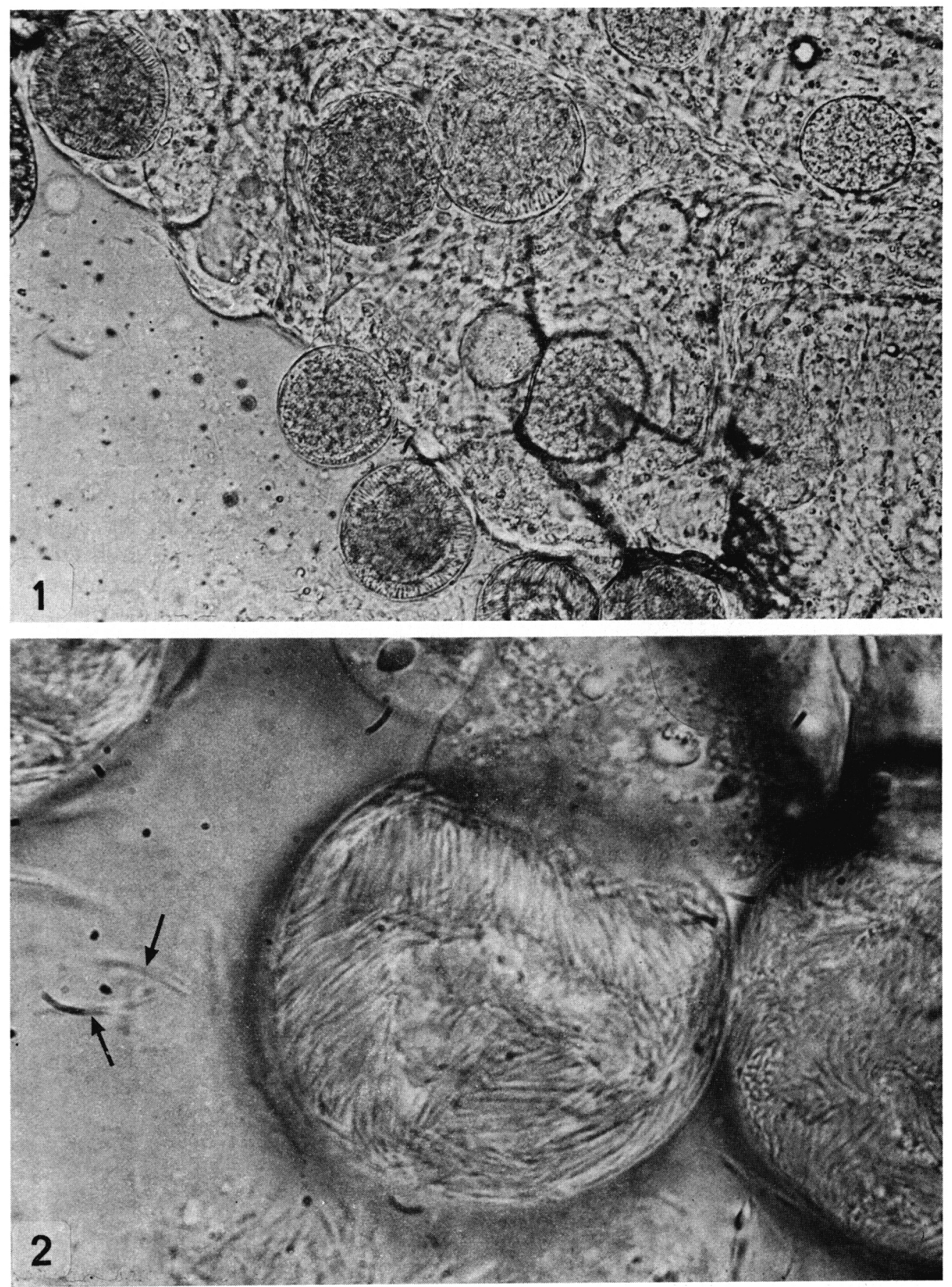

Fig. 1. - 1) Estomac d'Ánopheles stephensi avec des oocystes proches de la maturité ; 2) oocyste mûr et sporozoïtes (flèches) autour de l'estomac du Moustique (photo au contrastẹ interfẹ́rentiel). 
La longueur moyenne des sporozoïtes fixés au méthanol et colorés au Giemsa (fig. 2,2), mesurés au curvimètre sur dessins à la chambre claire, est de $8,5 \mu \mathrm{m}$ $\pm 0,12$.

\section{3 - Schizogonie pré-ÉRYthrocytaire}

Aucun schizonte pré-érythrocytaire n'a été trouvé dans la biopsie du Lemur macaco $39 \mathrm{CH}$. Rappelons que la biopsie a été effectuée 7 jours après la première inoculation de sporozoïtes et 5 jours après la seconde. Les premiers parasites avaient été détectés dans le sang le $9^{\mathrm{e}}$ après la première inoculation.

De nombreux schizontes ont été trouvés dans le foie à l'autopsie du Lemur fulvus pratiquée immédiatement après la détection des premiers parasites sanguins : $\mathrm{J} 9$ après la première inoculation et $\mathrm{J} 6$ après la seconde.

En coupes histologiques nous observons trois types morphologiques :

- type (a) (fig. 2,3). C'est le plus fréquent; les schizontes sont relativement jeunes, arrondis ou ovalaires, à contour souvent ondulé, mesurant en moyenne $30 \mu \mathrm{m}$ de diamètre. Les noyaux sont assez grands, granuleux, de forme grossièrement arrondie ; ils sont plus espacés que dans les stades plus âgés et régulièrement distribués dans un cytoplasme granuleux, pas très dense. La cellule hôte est légèrement hypertrophiée, plus claire que les cellules voisines. Son noyau, augmenté de volume, parfois déformé, présente une partie centrale claire, une chromatine condensée en périphérie et fréquemment un gros nucléole central ;

- type (b) (fig. 2, 4). Certains schizontes immatures sont de grande taille, allongés, ovalaires ou parfois lobés. Les mensurations de deux d'entre eux sont $33 \mu \mathrm{m} \times 60 \mu \mathrm{m}, 32 \mu \mathrm{m} \times 42 \mu \mathrm{m}$; leurs principales particularités sont : -- la forme et la disposition des noyaux, de grande taille et de forme irrégulière, souvent allongée ; ils se chevauchent et occupent pratiquement tout le volume du schizonte - le découpage nucléo-cytoplasmique en "pseudo-cytomères"; ils ressemblent beaucoup aux schizontes pré-érythrocytaire de Plasmodium falciparum et semblent présenter comme eux un processus d'aposchizogonie décrit par Bray (1960);

- type (c) (fig. 2, 5). Enfin, trois schizontes proches de la maturité ont été observés : ils sont grands $(47 \times 47 \mu \mathrm{m}, 25 \times 40 \mu \mathrm{m}, 33 \times 47 \mu \mathrm{m})$ avec souvent un ou deux lobes. Les noyaux sont petits et nombreux, le cytoplasme bleu pâle, granuleux. Le noyau de la cellule-hôte est hypertrophié, réduit à une couronne de chromatine et un gros nucléole.

Quelques zones d'infiltration macrophagique peuvent être observées, mais il n'y a aucun résidu de schizonte en voie de destruction.

Sur frottis par appositions, les schizontes forment des masses rondes très chromophiles, violet ou rouge foncé, dans lesquelles on devine les noyaux. Certains paraissent entourés par une sorte d'enveloppe rouge. Celle-ci n'est cependant pas visible en coupes. Une seule fois un schizonte de type (a) présentait sur coupes, quelques flaques de colloïde rouge dans le cytoplasme. 


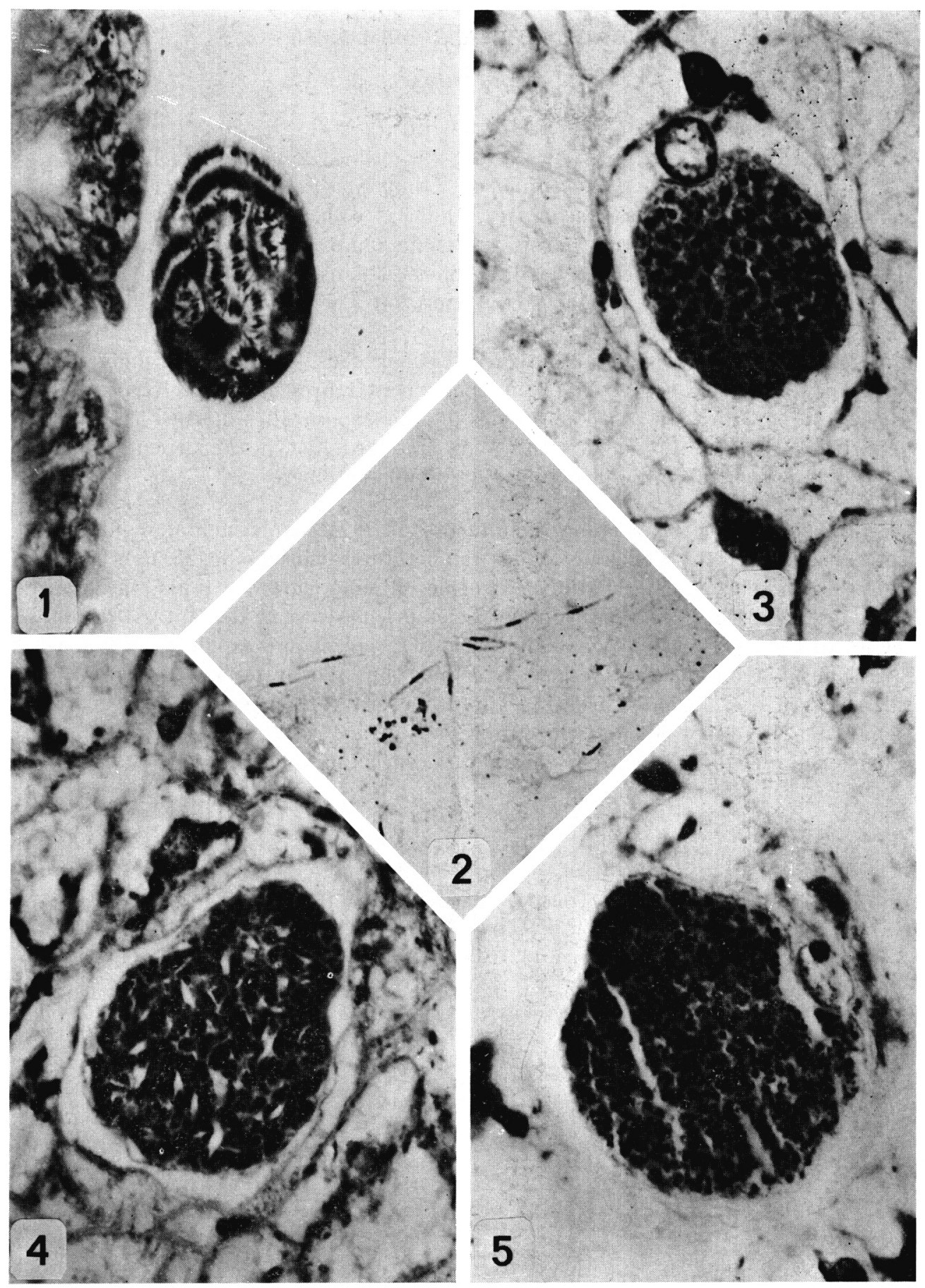

FIg. 2. - Oocyste mûr dans une coupe d'estomac d'Anophèle; 2) sporozoïtes dans un frottis de glandes salivaires coloré au Giemsa ; 3) schizonte pré-érythrocytaire de type $(a)$; 4) schizonte de type $(b) ; 5)$ schizonte de type $(c)$. 


\section{Discussion}

Les trois espèces de Plasmodium du Lemur macaco macaco sont transmises au laboratoire par Anopheles stephensi (Landau et coll., 1989).

Cependant elles ont été transmises en même temps et il nous est donc impossible de différencier les espèces, chez l'Anophèle et dans le foie du Lémur.

Les mesures de taille des oocystes et sporozoïtes ne permettent pas de détecter des populations différentes.

Par contre nous pensons pouvoir identifier deus types de schizontes exoérythrocytaires, le type $(a)$ et $(c)$ qui pourraient représenter deux stades de la mème espèce, l'un $(c)$ formé à partir des premiers sporozoïtes inoculés, l'autre $(a)$ à partir des sporozoïtes inoculés trois jours plus tard. Le type $(b)$ par contre paraît bien différent en raison de la présence de pseudo-cytomères.

En conclusion, cette étude préliminaire du cycle expérimental montre que la transmission peut s'effectuer à des températures voisines de $25^{\circ} \mathrm{C}$ et que la durée de la schizogonie hépatique est égale ou supérieure à 9 jours. Ce dernier caractère rapproche les Plasmodium de Lémuriens de ceux des autres Primates et les distingue des Plasmodium de Muridés à schizogonie rapide (2 à 3 jours).

Nous n'avons pas pu déceler de populations d'oocystes ou de sporozoïtes distinctes et les schizontes hépatiques n’ont pu être rattachés aux espèces correspondantes. Le dimorphisme (schizontes avec ou sans cytomères) semble indiquer l'existence de différences morphologiques spécifiques.

\section{RÉFÉRENCES}

Bray R. S. : Observations on the cytology and morphology of the mammalian malaria parasites. I. A process of apparent plasmotomy in the pre-erythrocytic phase of Laverania falcipara. Riv. Parassitol., 1960, 21, 267-276.

Landau I., Lepers J.-P., Rabetafika L., Baccam D., Peters W., Coulanges P. : Plasmodies de Lémuriens malgaches. Ann. Parasitol. Hum. Comp., 1989, 64, 171-184.

Lepers J.-P., Rabetafika L., Landau I., Peters W. : Une nouvelle espèce plasmodiale chez un Lémurien : Plasmodium coulangesi n. sp. Ann. Parasitol. Hum. Comp., 1989, 64, 163-170.

Shute P. G., Maryon M. : Laboratory techniques for the study of malaria. J. and A. Churchil, Livingstone, 1966, London. 